\title{
Controlled Environments from Sample Preparation to Electron Microscopy Characterization
}

\author{
C.S. Bonifacio ${ }^{1}$, P. Nowakowski ${ }^{1}$, K. Costello ${ }^{2}$, M.L. Ray ${ }^{1}$, R. Morrison ${ }^{2}$ and P.E. Fischione ${ }^{1}$ \\ ${ }^{1 .}$ E.A. Fischione Instruments Inc., Export, PA, USA \\ ${ }^{2}$ Quorum Technologies Ltd., East Sussex, UK \\ * Corresponding author: cs_bonifacio@fischione.com
}

Transmission electron microscopy (TEM) is a critical analytical technique in materials science, life sciences, and geology research. Currently, Li-ion batteries and catalysts, which are highly reactive and sensitive to air, are of interest because of their renewable energy potential [1-2]. A critical requirement in the development of these materials for tangible applications is the ability to characterize the materials in a pristine state, without environmental modifications or contamination. Preparation of batteries and catalysts involve pretreatment steps to remove surface oxides - which typically occurs in tools other than the tool used for analysis. Sample preparation and experimentation become challenging because it is necessary to transfer samples between milling systems and microscopes while simultaneously maintaining the material's integrity. Here we present robust controlled environments that protect the material from the sample preparation phase (bulk to focused ion beam [FIB] preparation) and through the electron microscopy characterization phase. High-quality Li-ion battery and supported-metal catalyst TEM specimens that are free from amorphous and Ga damage will be prepared and investigated.

Figure 1 shows a controlled environment workflow. A cross-section sample was loaded onto a scanning electron microscopy (SEM) shuttle (Fig. 2a) for broad ion beam (BIB) $\mathrm{Ar}^{+}$milling to remove surface oxides. A vacuum/inert gas transfer capsule [Fischione Instruments] (Fig. 2b) protected the sample postBIB milling. A glove box with a positive pressure environment was used to transfer the SEM shuttle from the vacuum/inert gas transfer capsule to a FIB transfer system [Quorum Technologies] (Fig. 2c), and thereafter, to a FIB system [Thermo Fisher Scientific] for TEM specimen preparation.

A TEM half grid was secured in a vacuum transfer specimen holder [Fischione Instruments] cartridge (Fig. 2d) and the cartridge was mounted on a TEM shuttle (Fig. 2e) and inserted into the FIB by means of the FIB transfer system. A TEM specimen was prepared using standard lift-out methods and polishing steps $(30 \mathrm{kV}$ and $5 \mathrm{kV})$. The shuttle was loaded into the FIB transfer system and then moved to the glove box. Subsequently, the cartridge was transferred from the TEM shuttle and mounted on the vacuum transfer specimen holder within the glove box. The TEM specimen holder was then moved to a concentrated ion beam $\mathrm{Ar}^{+}$milling system. $\mathrm{Ar}^{+}$milling was performed by rastering the beam within a defined area of the TEM specimen at decreasing milling energies. TEM, energy dispersive X-ray spectroscopy (EDS), and electron energy loss spectroscopy (EELS) were performed before and after ion milling to quantify the removal of FIB-induced damage and the robustness of the workflow's ability to prevent specimen oxidation and contamination. Controlled environment sample preparation $\left(\mathrm{Ar}^{+}\right.$and $\mathrm{Ga}^{+}$) and specimen characterization (EDS, TEM, and APT) experiments are underway.

References:

[1] B Diouf and R Pode, Renewable Energy 76 (2015), p. 375.

[2] J Gong and R Luque, Chemical Society Reviews 43 (2014), p. 7466. 


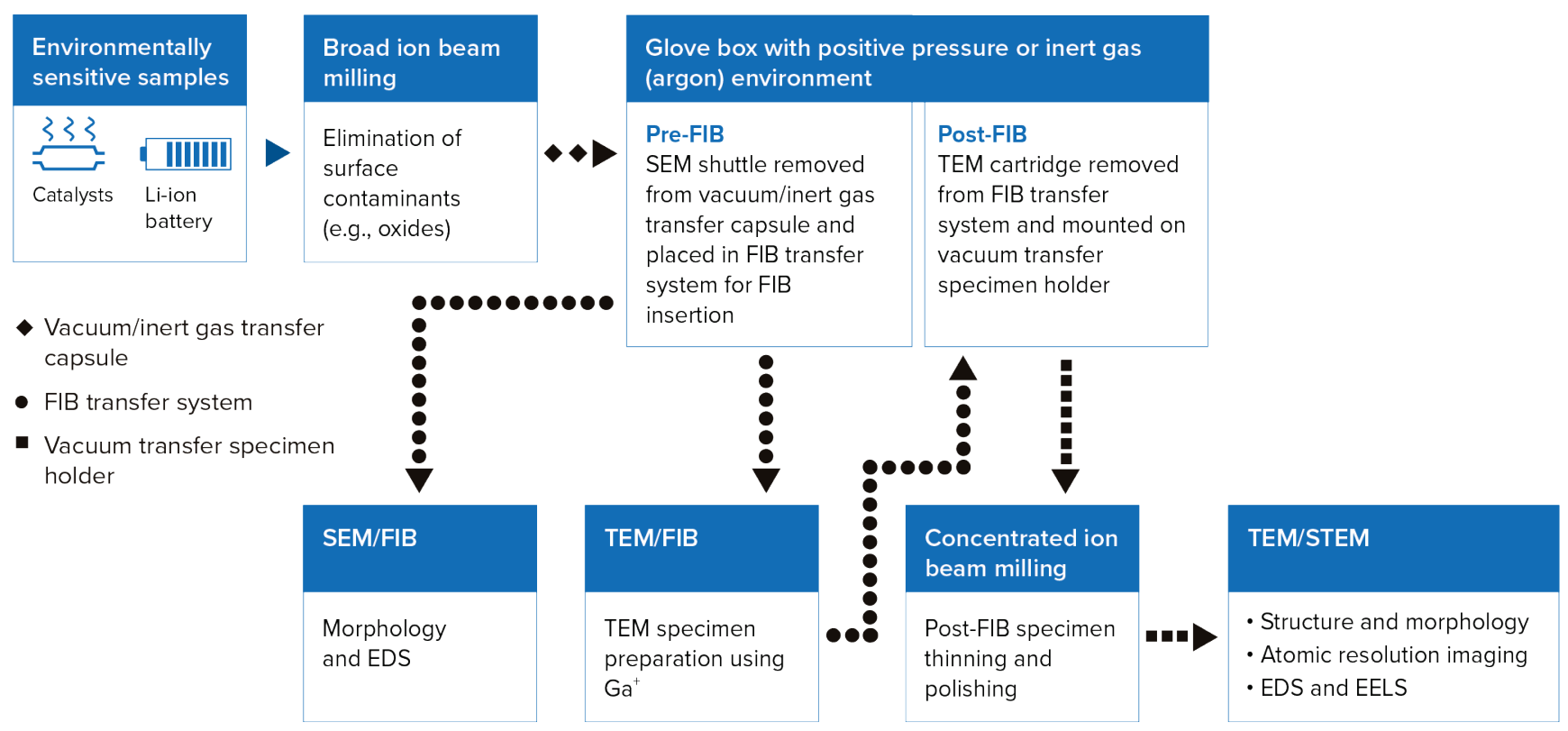

Figure 1. Controlled environments workflow for SEM and TEM sample preparation and microscopy characterization.

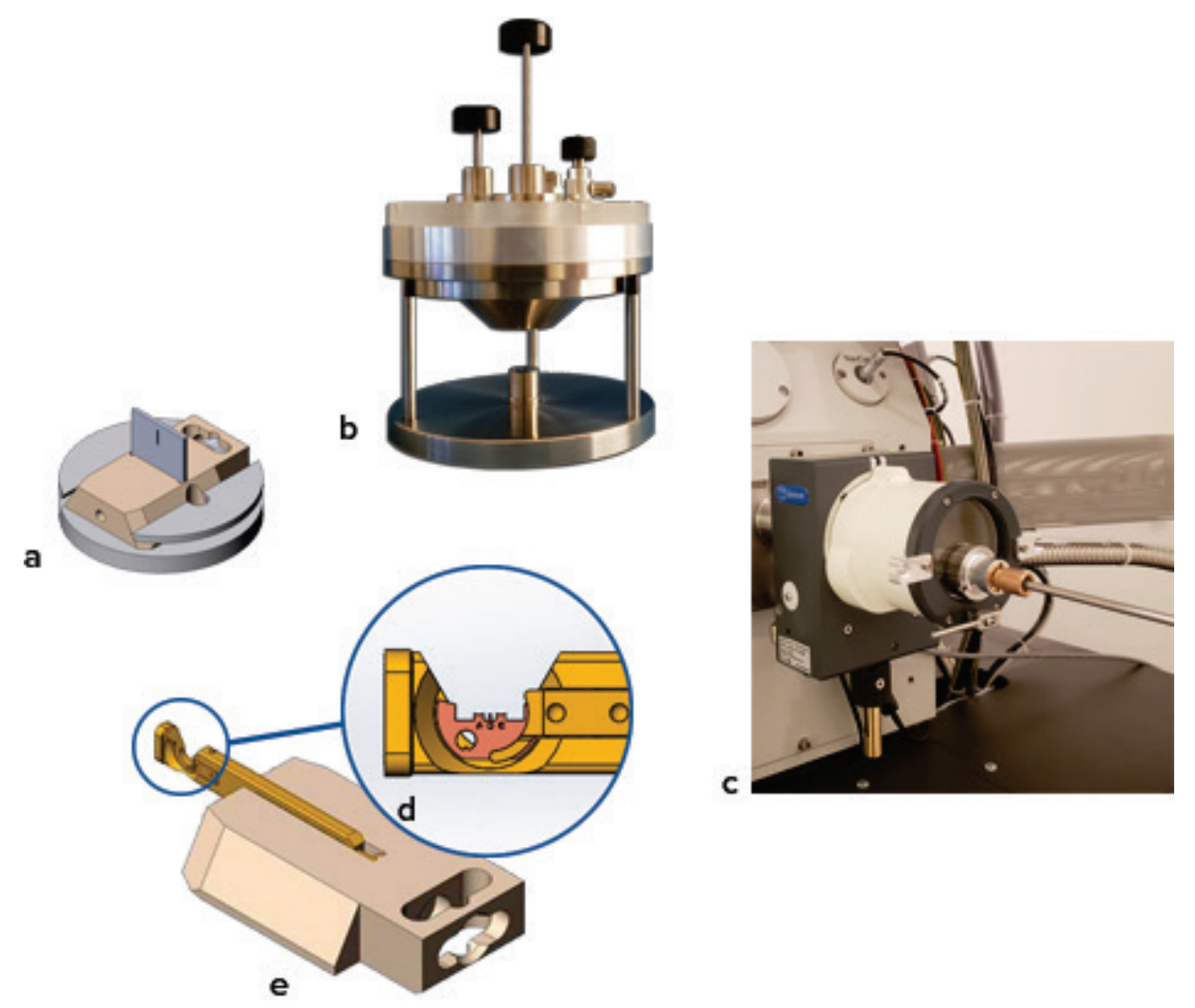

Figure 2. Controlled environments transfer components, clockwise from left: (a) SEM shuttle for broad ion beam (BIB) milling; (b) vacuum transfer capsule for transfer of BIB-milled sample to glove box; (c) FIB transfer system mounted on FIB; (d) TEM half grid mounted on vacuum transfer specimen holder TEM cartridge; and (e) TEM cartridge mounted on TEM shuttle. 